\title{
Culturas juveniles como perspectiva para analizar juventudes (1993-2018)
}

Carles Feixa ${ }^{1}$

\section{RESUMEN}

Este artículo de revisión traza un panorama de los estudios sobre las culturas juveniles latinoamericanas en los últimos veinticinco años, en un viaje que va del siglo XX al XXI (de la Generación X a la Generación @), con un prólogo en la Generación de las Tribus y un epílogo en la Generación Indignada. De los estudios pioneros sobre bandas y tribus urbanas, a los estudios más recientes sobre redes y culturas juveniles transnacionales, las expresiones culturales juveniles constituyen una óptica privilegiada para analizar las juventudes, sus continuidades, sus cambios y su contribución a la evolución social, política y cultural de las sociedades latinoamericanas en su conjunto.

Palabras clave: Culturas juveniles, América latina, Generación de las Tribus, Generación X, Generación $a$, Generación Indignada

1 Doctor en Antropolología Social (Universidad de Barcelona). Académico en la Universitat Pompeu Fabra (Cataluña-España). Miembro del grupo de investigación sobre Juventud, Sociedad y Comunicación: JOVIS.com. Coordinador de la Red de Estudios sobre Juventud y Sociedad - REJS. Correo electrónico: carles.feixa@upf.edu.

* Este texto se basa en los resultados de varios proyectos de investigación, el último de los cuales ha recibido financiación del European Research Council (ERC) en el marco del programa de Investigación e Innovación de la Union Europea HORIZON 2020, Grant Agreement Nº 742705. 


\title{
CULTURAS JUVENIS COMO PERSPECTIVA PARA ANALISAR AS JUVENTUDES (1993-2018)
}

\section{RESUMO}

Este artigo de revisão traça uma visão geral dos estudos sobre as culturas juvenis da América Latina nos últimos vinte e cinco anos, em uma viagem que vai do século XX ao século XXI (da Geração X à Geração @), com um prólogo na Geração das Tribos e um epílogo na Geração Indignada. Dos estudos pioneiros sobre bandas e tribos urbanas, até os estudos mais recentes sobre redes e culturas juvenis transnacionais, as expressões culturais juvenis constituem uma perspectiva privilegiada para analisar as juventudes, suas continuidades, suas mudanças e sua contribuição para a evolução social, política e cultural das sociedades latino-americanas em seu conjunto.

Palavras-chave: Culturas juvenis, América latina, Geração das Tribos, Geração X, GeRAÇÃOQ, GERAÇÃo INDIGNADA

\section{YOUTH CULTURES AS A PERSPECTIVE TO ANALYZE YOUTHS (1993-2018)}

\begin{abstract}
This review article traces an overview of the studies on Latin American youth cultures over the past twenty-five years, from the 2oth to the 2ist century (from Generation X to Generation @), with a prologue in the Generation of Tribes and an epilogue in the Indignant Generation. From the pioneering studies on urban gangs and tribes to the most recent studies on transnational youth networks and cultures, youth cultural expressions offer a privileged perspective to analyze youths, their continuities, changes and contribution to the social, political and cultural evolution of Latin American societies as a whole.

Keywords: Youth Cultures, latin America, Generation of Tribes, Generation X, Generation $a$, InDIGNANT GENERATION
\end{abstract}




\section{Introducción}

Cuando nació la revista Última Década, en r993, yo había cumplido zo años y acababa de regresar de México, donde había pasado un año investigando a los chavos banday había tenido ocasión de conocery trabar amistad con losjuvenólogos mexicanos (y) más tarde, a través de ellos, con los latinoamericanos). Ahora que esta revista tan relevante para la investigación y la acción en el mundo de los jóvenes cumple 25 años (ahora que se hace mayor), yo cumplo mis 55 años habiendo recorrido la mayoría de países iberoamericanos, habiendo conocido a sus más insignes juvenólogos, habiendo leído y publicado en las revistas sobre la juventud de la región, habiendo escuchado las vidas de jóvenes allíy aquí, siguiendo a los que emigraron del campo a la ciudady de América Latina hacia los Estados Unidos y más tarde hacia Europa, habiendo observado a jóvenes indígenas, rurales, urbanos, punketas, okupas, makineros, raperos, floggers, reyesy reinas latinos.

En los últimos años he publicado cuatro artículos en colaboración en la revista Última Década, que de algún modo reflejan la evolución temática y metodológica de la revista, que Klaudio Duarte analizó en su tesis doctoral (Duarte, 20I5). El primero, en colaboración con una socióloga italiana, trataba sobre el concepto de generación en las teorías sobre la juventud (Leccardi \& Feixa, 20II); el segundo, en colaboración con varios colegas mexicanos y chilenos, versó sobre la transición de la generación @ a la generación X (Portillo, Urteaga, González, Aguilera \& Feixa, 2012); el tercero, en colaboración con comunicólogas de mi actual universidad, trató sobre la generación indignada (Fernández-Planells, Feixa \& Figueras, 20I3); el cuarto, en colaboración con economistas de mi antigua universidad, trató sobre juventud y confianza política en el área mediterránea (Cabasés, Feixa \& Civit, 2015).

En este texto recupero algunos de estos estudios para trazar un panorama de las culturas juveniles latinoamericanas en las dos últimas décadas, en un viaje que va del siglo XX al XXI (de la Generación X a la Generación @), con un prólogo en la Generación de las Tribus y un epílogo en la Generación Indignada. Se trata, pues, de un artículo de revisión bibliográfica, que intenta poner en conexión la evolución de los estudios sobre culturas juveniles en América Latina con la trayectoria del propio autor. Cada apartado corresponde a un período y a una determinada conceptualización de las culturas juveniles. El texto está redactado en forma de ensayo, con referencias a las obras más significativas a pie de página y en la bibliografía final. ${ }^{2}$

2 Algunos párrafos fueron escritos originalmente en colaboración con el antropólogo chileno Yanko González, a quien agradezco sus aportaciones. 


\section{La Generación de las Tribus ${ }^{3}$}

Es posible que losjóvenes intenten constituir una especie de sub-cultura adolescente casi como una identidad definitiva, siendo por definición la condición juvenil algo transitorio.

(Enzo Faletto, I986)

En ig85 la Unesco declaró el Año Internacional de la Juventud: era un signo que las cosas no acababan de ir bien en el mundo de los jóvenes. El incremento galopante de la desocupación juvenil, el hundimiento de las ideologías contraculturales, el retorno a la dependencia familiar, generan discursos que ya no inciden en la capacidad revolucionaria y constructiva de los jóvenes, sino en la incertidumbre cultural y en los problemas que encuentran en la inserción social. Nace una actitud entre cínica y desencantada, que tiene múltiples traducciones en los imaginarios juveniles, pero que casi siempre guardan relación con una $\mathrm{K}$ subcultural: punKs, oKupas, sKinheads, maKineros. El sociólogo francés Michel Maffesoli (I990) hablará del "tiempo de las tribus" para a referirse a esta proliferación de microculturas juveniles, nacidas de la cultura de consumo o de los márgenes contraculturales, que ocupan nichos diferentes en el territorio urbano. Se trata de una metáfora perfectamente aplicable a las culturas juveniles del fin del siglo XX, fruto de la confluencia de comunidades hermenéuticas donde fluyen los afectos y se actualiza lo "divino social", caracterizadas por reafirmar las fronteras estilísticas, las jerarquías internas y las oposiciones frente al exterior. Sin embargo, es mucho más difícil de aplicar a los estilos juveniles emergentes en este cambio de milenio, que más que las fronteras enfatizan los pasajes, más que las jerarquías remarcan las hibridaciones, y más que las oposiciones resaltan las conexiones. Vivir la juventud ya no es -como en el complejo de Tarzán- transitar de la naturaleza a la cultura, ni tampoco -como el complejo de Peter-Pan-, resistirse a la adultez, sino experimentar la errancia del destino incierto, como en el complejo de Replicante, tomado del humanoide de Blade Runner, que se rebela porque no tiene memoria del pasado. Se trata, quizá, de una de las manifestaciones exteriores de eso que se le ha llamado el "fin de las ideologías" y el "fin de la historia" (conviene recordar que la década acabó con la caída del muro de Berlín y del comunismo soviético).

En I983 la misma UNESCO publicó un informe titulado "La juventud en la década de los 80 ", en el cual se dejaba claro los términos en que se tenía que plantear el debate: "Ni el enfoque ni el lenguaje característicos de la década de los 6o' parecen adaptarse a las nuevas realidades que la juventud tendrá que afrontar en la década que empezamos. En el ' 68 se hablaba de confrontación, protesta, marginalidad, contracultura... en definitiva, era un lenguaje que denotaba una confianza posible en un cambio hacia un mundo mejor. Tal vez en el próximo decenio las palabras claves que experimentarán los jóvenes

3 Ver Feixa (1998, 1998b); Reguillo (1991); Margulis (1997); Pérez-Islas (2006); Valenzuela (1998). 
serán: paro, angustia, actitud defensiva, pragmatismo, incluso súpervivencia" (I983, p. II). En este contexto, ¿es legítimo pensar en la juventud como una categoría social dotada de una cierta unidad de representaciones y actitudes? La respuesta de Pierre Bourdieu es aparentemente lapidaria: "La jeunesse n'est qu'un mot" (I990). Con esta frase Bourdieu quería decir que la edad no es más que una forma de marcaje social que se aplica en grupos en competencia -"jóvenes" y "viejos"- para grabar simbólicamente su preeminencia actual o futura; el análisis científico de las edades no es irrelevante, pero se limita al estudio de las luchas para la clasificación.

En América Latina, a la continuidad de varias dictaduras, se sumó durante los 8o' intensas crisis económicas, que rotularon a estos años como "la década perdida". Deudas externas impagables, grandes déficits fiscales y volatilidades inflacionarias y de tipo de cambio, devastaron materialmente al continente, ensañándose, particularmente con las y los jóvenes (desempleo y exclusión social). "Únanse al baile de los que sobran, nadie nos va a echar de más, nadie nos quiso ayudar de verdad" cantaba el grupo chileno de rock latino "Los Prisioneros" en la primera mitad de los 8o', describiendo a un enorme contingente juvenil de raíz urbano-popular que el sistema los había dejado -como continúa la misma canción- "pateando piedras", lírica que se amplifica en el aserto punketa mexicanizado "la neta no hay futuro". La especificidad de estos actores estribaba en su condición de marginados por un sistema político (dictaduras), económico (paro, carestía) y social (marginación y estigmatización) y por su capacidad de aglutinación y creación de referentes comunes de resistencia: unos a partir de una lenta imbricación con movimientos de base poblacional (barrial), religiosos, políticos o estudiantiles de izquierda; otros, paralizados por la exclusión -con altos índices de consumo de drogas y alcohol- y los menos, pero posteriormente en ascenso progresivo, articulados a las acciones y producciones contraculturales situadas alrededor de las culturas juveniles operantes al interior de los segmentos urbano-populares, como metaleros -heavis-, neohippies militantes/revolucionarios o jipitecas, punks, newwaves, chavos bandas, entre otros.

La extensión y amplitud de la juventud urbano-popular en la región se había fraguado -con sus variantes- por las políticas estatales de promoción popular en los 6o' y parte de los 70' con la aparición de urbanizaciones, poblaciones y extensas barriadas que acogieron la migración campo-ciudad. El desajuste estructural entre el sistema educativo y el sistema laboral provocó la discontinuidad de la incorporación de las nuevas generaciones al mundo adulto y, por lo tanto, amplió esta agregación juvenil en forma exponencial, obligando a las y los jóvenes de la época a padecer y permanecer como jóvenes. Esta falta de oportunidades de inserción adulta, se vieron agudizados tanto por el capitalismo dependiente, como por las situaciones coyunturales de crisis nacionales. Hasta el año Internacional de la Juventud proclamado por la ONU en 1985 , la gran mayoría de los estudios socioculturales en América Latina habían tendido a ignorar la dimensión generacional: tanto los estudios sobre las comunidades 
indígenas, como los centrados en sociedades rurales y urbanas, tendieron a ver a sus sujetos de estudio como indios, campesinos, colonos, hombres, mujeres, burgueses, obreros, pero no como niños y niñas, y todavía menos como adolescentes o jóvenes. La explicación tradicional a estas omisiones pone énfasis en la inexistencia de las categorías de infancia y juventud en las sociedades latinoamericanas más allá de algunas minorías sociales (clases medias) y territoriales (zonas urbanizadas). Este supuesto se basa en la concepción de que la gran mayoría de latinoamericanos pertenecientes a los sectores subalternos tienen una temprana incorporación a la vida adulta. En la segunda mitad de la década de los años ochenta, estas omisiones sociohistóricas y precariedades teóricas comenzaron progresivamente a resolverse. Aquí, tanto la sociología como la psicología se abren en forma incipiente a nuevos paradigmas teóricos y epistemológicos, que tienen que ver con el progresivo desarrollo de las ciencias sociales en América Latina, lo que posibilita una diversificación considerable en las aproximaciones hacia la realidad juvenil, transitando desde perspectivas modernizadoras marxistas y el énfasis en los movimientos sociales, hasta perspectivas más culturológicas y subjetivas.

De manera simultánea en varios países latinoamericanos la reflexión sobre los jóvenes se trasladó al terreno de las bandas y las culturas juveniles, de los territorios nómadas desde los que los jóvenes de la periferia social y territorial accedían al centro del escenario. Por ello, al terminar la década, algunos referentes de la escuela latinoamericana de estudios culturales (como García Canclini, Martín-Barbero, Monsiváis y Sarlo) plantearon reflexiones teóricas e históricas sobre la juventud de amplio alcance, basándose en estudios de campo sobre las culturas juveniles llevados a cabo por nuevas generaciones de investigadores. De este modo, los estudios sobre la juventud pasaron de ocupar un lugar marginal a un lugar importante en los debates de las ciencias sociales, convergiendo (a veces de manera espontánea) con las teorías europeas en boga durante la misma época, como los estudios subculturales de la Escuela de Birmingham, la teoría de la distinción de Bourdieu y el tribalismo de Maffesoli.

\section{La Generación $X^{4}$}

P. ¿Cómo ve el panorama?

R. Como una luchay una disputa entre un reloj que chequea el horario de ingreso de los empleados de una empresa, que es el reloj de Fox, y el nuestro, que es un reloj de arena. La disputa es entre que nosotros nos acomodemos a ese reloj de chequeo y Fox se acomode al reloj de arena. No va a ser ni lo uno ni lo otro. Tenemos que entender, ély nosotros, que tenemos que construir otro reloj de común acuerdo...

(El País, 25 de marzo de 200I)

4 Ver Feixa (2006); Feixa, Portillo, Urteaga, González, Aguilera (2012); Reguillo (1998); Valenzuela (1998). 
En 200 el escritor colombiano Gabriel García Márquez entrevistó al subcomandante Marcos, poco después de que éste entrase triunfante en el Zócalo -la plaza central de la ciudad de México- tras recorrer todo el país seguido por su pequeño ejército de jóvenes indígenas, con el apoyo de numerosos jóvenes universitarios. Unas frases del líder zapatista llamó particularmente la atención. Ante la pregunta sobre las perspectivas de las negociaciones de paz en Chiapas, Marcos respondía comparando el reloj de arena del tiempo indígena con el reloj mecánico del tiempo de la modernidad. Cuando García Márquez le preguntó por qué llevaba una linterna, un aparato de comunicaciones y un reloj en cada mano, su respuesta fue clarividente: "Con un reloj llegué a la selva y el otro es de cuando empezó el alto al fuego. Cuando las dos horas coincidan significa que se acabó el zapatismo como ejército y que sigue otra etapa, otro reloj y otro tiempo".

En América Latina, la revuelta zapatista que emergió en Chiapas en enero de 1994, liderada por un viejo líder estudiantil al día de las nuevas tecnologías, protagonizada por un puñado de jóvenes indígenas, apoyada más tarde por jóvenes urbanos y universitarios de México y de otros países del subcontinente, acabó gozando de las simpatías de jóvenes de muchos otros países, que se apropiaron de ella como su "revolución generacional" (de la misma manera que la revolución cubana o la sandinista fueron emblemas para otras generaciones). Mostró la potencialidad de los jóvenes que se involucraron en esta revuelta. Se instalaron, sin pensarlo siquiera, en la primera movilización global que usó a las tecnologías de la información como ninguna otra antes. Este movimiento ilustra muy bien la forma en que la Gen X tuvo que lidiar con el advenimiento de Internet.

La revuelta zapatista, más que las armas, utilizó las nuevas tecnologías de la comunicación para difundir sus consignas. Lo que algunos han llamado "la primera guerrilla postmoderna" se convirtió en un referente generacional para aquellos jóvenes que habían entrado en la juventud con la caída del muro de Berlín. En 1999, en Seattle, la década se cierra con una protesta contra una reunión de los poderes económicos mundiales, protagonizada por el llamado Movimiento de Resistencia Global. Paradójicamente, los "antiglobalizadores" son los primeros en utilizar las nuevas tecnologías de la era de la globalización. Unos años antes, en I991, el escritor Douglas Coupland había popularizado el término "Generación X" para referirse a una juventud marcada por las incertidumbres y las paradojas de la sociedad postmoderna, y por la falta de un sistema de valores sólido. Pero no es hasta la segunda mitad de la década cuando esta expresión empieza a aplicarse a movimientos juveniles de nuevo signo que surgen en América Latina, lo que coincide con la emergencia de las culturas juveniles como nodo de los estudios culturales latinoamericanos (García Canclini, 2004).

Finalizado el siglo XX, la agenda latinoamericana de los estudios sobre la juventud está por cimentarse. Tras una fase ensayística-especulativa en el 
primer tercio del siglo, una fase empírico-modernizadora en los años sesenta y setenta, y una fase etnográfico-experimental después de 1985 , es necesario un esfuerzo de reformulación teórica y conceptual que contribuya a resituar la investigación de campo sobre bases aún más sólidas. Aunque durante la década de los 9o> se evidencia un desarrollo articulador mayor, como consta en los trabajos de Rossana Reguillo; las «Reuniones Nacionales de Investigadores sobre Juventud», en México D. F.; las contribuciones de diferentes investigadores sobre juventud Latinoamericana realizada por la Universidad Central de Colombia, publicado en Viviendo a toda (Cubides, Laverde \& Valderrama, 1998), la revista Última Década en Chile, lo cierto es que aún hay temas axiales a dilucidar: la construcción histórica y cultural de la juventud a partir de la diversidad; la relectura y producción teórica sobre las generaciones en una óptica latinoamericana; y la metamorfosis de la juventud en la era de la globalización.

\section{La Generación $a^{5}$}

\section{Al preguntarse por lajuventud, la sociedad trata de saber cómo comienza su futuro (Néstor García Canclini, 2004)}

Si la última generación del siglo XX fue bautizada por el término "generación X", podemos bautizar a los jóvenes que transitan al siglo XXI como a la generación @. Huelga decir que las generaciones no son estructuras compactas, sino solo referentes simbólicos que identifican vagamente a los agentes socializados en unas mismas coordenadas temporales. Desde esta perspectiva, el término pretende expresar tres tendencias de cambio que intervienen en este proceso: en primer lugar, el acceso universal -aunque no necesariamente general- a las nuevas tecnologías de la información y de la comunicación; en segundo lugar, la erosión de las fronteras tradicionales entre los sexos y los géneros; y en tercer lugar, el proceso de globalización cultural que conlleva necesariamente nuevas formas de exclusión social a escala planetaria. Al bautizar a los jóvenes de hoy como "generación @”, no pretendemos postular la hegemonía absoluta del reloj digital (o de la concepción virtual del tiempo). Lo que pretendemos resaltar es el papel central que en esta transformación tienen las concepciones del tiempo en los jóvenes, que impacta la propia configuración del espacio social en el cual la juventud actúa. Por esos procesos, se reactualizan los modos de estar juntos y, dentro de ello, las modalidades de consumo cultural. El consumo de bienes audiovisuales es seguramente el sector del mercado que más claramente refleja estas tendencias de cambio.

De ahí que en América Latina constatemos una serie de empalmes; es de-

5 Ver Feixa (2000), Feixa \& González (2004); Feixa, Porzio \& Recio (2006); Chaves (2010); Urteaga (2011). 
cir, resabios de la generación X presentes en los jóvenes de inicios del Siglo XXI, así como marcas de la Generación @ en los jóvenes que tuvieron que "subirse al carro" de la superautopista de la información en plena efervescencia de su juventud. Los contextos sociopolíticos y económicos de la región obligan a problematizar las formas en que la Generación X tomó forma en este continente. No podemos soslayar, en este sentido, que fue hija de una generación que sufrió las dictaduras políticas que azotaron a los distintos países latinoamericanos y que por supuesto impactaron de manera específica a los sujetos que crecieron en esa época de terror y barbarie. Los padres de la Gen X tuvieron que migrar, exiliarse y en muchos casos, fueron sistemáticamente desaparecidos. En tanto los procesos de dictadura dieron paso a transiciones a la democracia de distinta intensidad en los países de la región y a profundas crisis económicas, la juventud apareció marcada como problema social, apática en lo relativo a lo político y como una gran incógnita respecto a su comportamiento futuro.

La producción de publicaciones colectivas o incluso películas, se basa en esta tradición de la que algunos de nosotros hemos estado activos "instigadores" durante casi tres décadas, y que une el trabajo realizado en la península Ibérica y los países de América Latina. La aparición de esta tradición intelectual se expresa en las redes sociales académicas basadas en intercambios personales y virtuales, con experiencia en conferencias, afinidades teóricas y compromisos políticos (Reguillo y Feixa, 2004). Su origen se remonta a través de varias redes de investigación nacionales y transnacionales, revistas académicas y libros editados. En cuanto a las redes, fueron promovidas por las agencias estatales (como la Red Mexicana de Investigadores en Juventud, en la década de r99o), por las nuevas generaciones de investigadores jóvenes (como la Red Argentina de Investigadores Juventudes, en la década de 2000), o por las agencias de cooperación de investigación (como el Grupo de Trabajo Juventud de CLACSO, Consejo Latinoamericano de Investigaciones Sociales). En cuanto a las revistas, la consolidación de un campo científico se debe a tres revistas específicas, distribuídas en toda la región: Ultima Década en Chile (desde 1993), Jóvenes en México (entre 1996 y 2006) y la Revista Latinoamericana de Ciencias Sociales, Niñezy Fuventud en Colombia (desde 2003). Numerosos libros editados son el resultado de las conferencias, coleccionar obras de los principales actores en el campo, Aproximaciones a la Diversidad de lo juvenil (Medina, 200o); Fóvenes, Culturas e identidades Urbanas (Nateras, 2002); Pachucos, malandros, punketas (Feixa et al, 2002); Grafitis, Grifotas, Okupas (Feixa et al, 2002); Fovens na América Latina (Caccia Bava et al, 2004); Fóvenes, Culturay Política en América Latina (Alvarado y Vommaro, 2010), entre otros. En todo este recorrido, es indudable el papel jugado por la OIJ; como impulsora de informes (Hopenhayn, 2007) y como instigadora de redes y encuentros. 


\section{La Generación Indignada ${ }^{6}$}

(Propugnamos) una sublevación de los jóvenes contra la juventud (...) Habíamos menospreciado el deseo de los jóvenes de ser adultos contra todo un entramado social, político y cultural que quiere retenernos en la infancia.

(Juventud sin Futuro, 20II)

A lo largo de 20iI, un fantasma ha recorrido Europa (y más allá): el fantasma de la indignación. Es un fantasma con múltiples caras, aunque la más visible tiene rostro juvenil. Apareció primero en la periferia de París y Atenas, acampó luego en el centro del Cairo, Lisboa, Madrid y Barcelona, y ha vuelto a irrumpir en Londres y Tel-Aviv, pero también en Santiago de Chile, São Paulo, Bogotá y Ciudad de México. Tras el fantasma, una presencia: la del nuevo lumpenproletariado de la era postindustrial, constituído por esos jóvenes hiperformados -e hiperinformados- y sin embargo precarizados, conectados a través de las redes sociales, que a veces reaccionan en forma creativa y pacífica (en forma de comedia) y otras en forma más airada y violenta (en forma de tragedia). Tras esta presencia inquietante, un espectro: el de una crisis económica global que afecta con particular intensidad a las nuevas generaciones, cuyos efectos van más allá de la precariedad material, presentándose en forma de crisis de valores (o más bien de valores de la crisis).

¿Qué tienen en común todos estos movimientos? ¿Cuáles son las extrañas galerías que conectan sus actores, motivaciones y propuestas? ¿Qué lecciones plantean a nuestras sociedades democráticas? Hace un tiempo propuse entonces considerar tres modelos de juventud alternativos que convivían en nuestra sociedad: el de Tarzán o niño salvaje, el de Peter Pan o eterno adolescente y el del Replicante o joven androide. Entre la criminalización y la domesticación del botellón (consumo de alcohol en la vía pública) -y de la propia juventud-, el texto acababa pronosticando "una tercera vía que trate a los jóvenes, no como replicantes, sino como ciudadanos capaces de reinventarse como actores sociales" (Feixa, 2009).

$\mathrm{El}$ actual ciclo de protestas juveniles, tanto las que surgen de las periferias urbanas como las que ocupan el centro de las ciudades, tanto las que nacen en Europa como las que lo hacen al sur del Mediterráneo y allende los mares, tanto las protagonizadas por estudiantes de clase media como las lideradas por subocupados y desocupados, no son revueltas de la miseria sino del bienestar. Están protagonizadas por una generación no ya educada en la ética puritana del ahorro, sino en la ética hedonista del consumo y, sobre todo, en la ética posmoderna de la Red (la nética). En este ciclo podemos distinguir dos prólogos, dos epílogos y algunos momentos culminantes.

Como prólogos, las revueltas callejeras en dos países europeos: el que in-

6 Ver Feixa (2010); Oliart \& Feixa (2012), Feixa, Fernández-Planells y Figueras (2004). 
ventó la democracia (Grecia) y el que la reinventó (Francia). En otoño de 2005 , en la revuelta de las banlieueus, una coalición de jóvenes blanc-black-beur puso en práctica lo que el filme La Haine había pronosticado: la conversión de la indignación en rabia, encendida por un abuso policial real o percibido, y dirigida contra algunos íconos de la sociedad de consumo: escaparates rotos y coches quemados (cabe recordar que en otras ciudades europeas como Berlin la quema de coches se han convertido en una especie de ritual que se repite periódicamente). En 2008, el otoño griego sirvió para dramatizar los efectos de la crisis financiera internacional, en forma de una revuelta protagonizada por jóvenes airados, educados para el estado del bienestar, pero que de repente descubrían la amenaza de un estado de malestar.

Como momento culminante, la primavera mediterránea de 20II, con la ocupación pacífica de las plazas. Primero, la protesta contra regímenes autocráticos impulsadas por la Generación Rai-Rap tunecina y egipcia, educada en Facebook más que en las escuelas coránicas o baazistas (una revuelta triunfante aunque sus jóvenes líderes hacktivistas hayan sido rápidamente fagocitados por políticos de más edad). Luego, la marcha impulsada en Portugal por la Geração a Rasca (la Generación en apuros), formada por los más afectados por

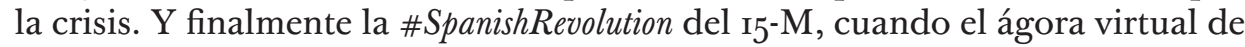
las redes sociales se convirtió en una acampada real. Aunque algunos la vieron al principio como un macrobotellón, la acampada despertó la simpatía ciudadana: la Generación Ni-Ni se convertía súbitamente en Generación Sí-Sí-Sí, pues además de estudiar y trabajar, a los jóvenes indignados le quedaba tiempo para comprometerse en un movimiento que atrajo la atención mundial y se diseminó por otros lugares donde no sobran motivos para la indignación, como México, en cuyos zócalos se han convocado en los últimos tiempos concentraciones de Indignados contra narcos y políticos corruptos.

Como epílogos, la revuelta de los suburbios ingleses del reciente verano, protagonizada por una coalición de jovenes "yob" (boy al revés), hijos de inmigrantes caribeños, africanos, asiáticos o de la clase obrera blanca, dependientes a su pesar del estado de bienestar, que pusieron en práctica lo que el filme Do the right thing había previsto: la revuelta del gheto multicultural, con una secuencia parecida a la de Francia (chispa policial, saqueo hiperconsumista y desprecio institucional), pero con algunas particularidades (como la participación de jovenes de la clase media alta). Y finalmente, la revuelta estudiantil en Chile, donde una nueva generación de "pingüinos" (el nombre que reciben los estudiantes de secundaria por su uniforme) ponen en jaque al gobierno neoliberal, la protesta universitaria en Colombia, prolegómeno del proceso de paz con el último grupo guerrillero en armas, y en México, donde el movimiento \#YoSoyı23 intentó evitar la vuelta al poder del PRI, promoviendo de paso el debate sobre la renovación de la democracia. 


\section{DE CULTURAS SUMERGIDAS A CULTURAS EMERGENTES}

En 2018 la revista Communication Theory publicó una special issue sobre los estudios de comunicación en América Latina, seleccionando para comentarlas dos obras de referencia: el libro de Eliseo Verón La semiosissocial, y el de Rossana Reguillo Emergencia de culturasjuveniles (2000), que tuve el honor de comentar junto con Mònica Figueras (Feixa \& Figueras, 20I8). La obra de Reguillo constituye de algún modo un parteagas de los estudios sobre culturas juveniles, siendo publicado en dos años emblemáticos. El original apareció en la Enciclopedia Latinoamericana de Sociocultura y Comunicación (Grupo Editorial Norma, Buenos Aires) en el año 2000, la división del milenio que amenazaba con restablecer no solo las computadoras que usan Windows, sino también el paso a la segunda modernidad. La edición actualizada fue publicada en 2012 por Siglo Veintiuno Editores, con un cambio significativo en el título -las culturas juveniles ya no eran "emergentes"- y con un nuevo prólogo y un nuevo capítulo que "busca ser la bisagra entre finales del siglo XX y comienzos del XXI" (citado en Scolari, 2013, par. 6). Esto fue poco después de que las plazas en muchas partes del mundo, se llenaron de protestas indignadas, y mientras \#YoSoyız 2 surgió en México como una profecía autocumplida de las teorías del libro sobre la capacidad estratégica de las culturas juveniles para comunicar las formas políticas de desencanto.

Reguillo actualizó el libro durante su etapa como chair de la cátedra Andrés Bello de la Universidad de Nueva York, donde pudo integrar su experiencia de años de estudio y trabajo de campo con grupos juveniles de diferentes países. Entre la primera y la segunda edición, se produjeron cuatro cambios relevantes: el avance de la crisis financiera internacional y sus consecuencias en los organismos juveniles; el avance de la sociedad de la red; el avance en los estudios de culturas juveniles (dirigido por el autor como homólogo latinoamericano de la Escuela de Birmingham); y el avance de los estudios culturales latinoamericanos. Este avance fue representado por dos autores muy referenciados con quienes Reguillo ha colaborado y entablado un diálogo durante años. Su trabajo entreteje los argumentos de Néstor García Canclini (1989) sobre la juventud como un escenario para las culturas híbridas emergentes y las reflexiones de Jesús Martín-Barbero (I998, 20I7) sobre las culturas juveniles como palimpsestos de identidad.

A partir de su etnografía pionera sobre las pandillas juveniles en Guadalajara (México), centrada en las formas de comunicación callejera (Reguillo, I99I), en Culturas juveniles, la autora propone un recorrido teórico-etnográfico organizado en cinco etapas: considerar cómo pensar la juventud, mapear la identidad, interpretar la biopolítica, conducir una etnografía de las culturas emergentes y repensar la ciudadanía de los jóvenes. Reguillo propone un análisis de las dimensiones biopolíticas integradas en el cuerpo, la materialidad en la que se anclan las identidades y prácticas juveniles y sobre qué poder se ejerce. 
Con este objetivo, en el capítulo sobre etnografía revisa casos clave de denominaciones de identidad definidas por los propios jóvenes a través de procesos de hibridación cultural: punks (fusión del anarquismo y la posmodernidad), taggers (fusión de lo local y global), raztecas (fusión del rap y neoindigenismo) y dilleis (versión mexicana de los disc jockeys, fusión de chamanismo y tecnología). En todos los casos va más allá de la cartografía de la estética, buscando los vínculos con la política a través de un enfoque original en relación con los estudios culturales anglosajones y argumentando que "no es posible imaginar la comunicación aparte de los procesos socioculturales y el reverso" (Reguillo, 2005, p.r97). Como ella dice en la introducción, el libro no trata solo sobre los jóvenes, sino también sobre la comunicación: "En sus estrategias, en sus formas de interacción comunicativa, en sus percepciones del mundo, hay un texto social esperando a ser descifrado: la de un tipo de política en minúsculas que puede hacer que el mundo, la localidad, el futuro y cada día sean un mejor lugar para vivir" (Reguillo, 2012, p.I5).

El libro es un análisis exhaustivo de los jóvenes y la cultura juvenil que evita el término "subcultura" debido a su matiz occidental y jerárquico; también es una reflexión profunda que no culpa a los jóvenes ni los idealiza. La autora cree que las culturas juveniles se manifiestan con expresiones, símbolos e idiomas para ocultar la esperanza y el miedo al mismo tiempo. Reguillo es muy honesta desde el principio, haciendo explícito ya en el primer capítulo su postura ideológica y sus implicaciones. Por un lado, el libro es un buen ejemplo de cómo estudiar un fenómeno en la intersección de diferentes disciplinas -sociología, antropología, historia y comunicación- con un fuerte componente crítico y compromiso político. También hace una contribución significativa como un modelo teórico-metodológico de cómo llevar a cabo un análisis sociocultural de un tema. A través de una profunda contextualización histórica y social, estudia el desconcierto de los jóvenes en el contexto social donde se insertan y las causas que provocaron este desconcierto. Acepta que las identidades juveniles están globalizadas, mientras que al mismo tiempo sostienen la especificidad histórica y cultural. Finalmente, proporciona un fundamento de la postura metodológica adoptada, la perspectiva interpretativa-hermenéutica que trasciende las posturas esencialistas "sin perder la centralidad de género, etnia y centralidad del territorio" (Reguillo, 20I2, p.37).

Asi pues, el libro de Reguillo describe la transición de la $\mathrm{I}^{\mathrm{a}}$ fase de los estudios sobre culturas juveniles (que podemos denominar "culturas sumergidas", subterráneas o underground, ejemplificadas en las monografías sobre bandas, pandillas y combos juveniles), a la $2^{\text {a }}$ fase (que podemos denominar "culturas emergentes", ejemplificadas en los afterpunks, taggers, raztecas y dilleis estudiados por la autora). Tras la publicación de la segunda edición del libro, en 2012, y coincidiendo con los movimientos indignados, hemos entrado en una $3^{\mathrm{a}}$ fase, en la que las culturas juveniles ya no son emergentes sino emergidas e incluso hipervisibles, lo que explica el boom de estudios sobre las mismas, en casi 
todos los países latinoamericanos, que no podemos reseñar aquí. El marco contextual de dicha fase es lo que he denominado Generación \# o Hashtag (Feixa, Fernández-Planells \& Figueras, 20I6), caracterizada por la consolidación del proceso de globalización, digitalización y comercialización de la cultura juvenil, así como por el uso político de las redes sociales, que la misma Reguillo ha analizado en su último libro, Paisajes insurrectos (Reguillo, 20I7).

En esta transición, hemos pasado de culturas juveniles apolíticas a culturas juveniles políticas o politizadas y a políticas de la cultura juvenil, es decir, a esfuerzos institucionales para comprender, controlar, encauzar o aprender de las culturas juveniles, que han dejado de ser un recurso lúdico para convertirse en el eje de las políticas públicas, tanto las de cooptación como las de supervisión o incluso las directamente juvenicidas (Aguilera, 20r6; Valenzuela, 20I6). Esa puede ser precisamente una de las funciones de las políticas de juventud: hacer resonar esas voces e incorporarlas al debate público. En otras palabras: se trata de conseguir que las nuevas expresiones culturales juveniles se conviertan en una herramienta para renovar la cultura democrática de todos y todas, para construir una ciudadanía para el siglo XXI.

\section{REFERENCIAS}

Alvarado, S.V. \& Vommaro, P.A. (Eds.) (2010). Fóvenes, culturay política en América Latina, 1960-200o. Buenos Aires: Homo Sapiens, CLACSO.

Aguilera, O. (2016). Movidas movilizaciones y movimientos: cultura política y políticas de las culturasjuveniles en el Chile de hoy. Santiago: RIL editores.

Bourdieu, P. (I 99o). La 'juventud' no es más que una palabra. Sociologíay cultura (pp. I63-I73). México: Conaculta-Grijalbo

Cabasés, M.A., Feixa, C., \& Civit, R. (2015). Jóvenes y confianza política en un contexto de desestabilización social e institucional: Un estudio comparativo en países de la Cuenca del Mediterráneo. Última Década, 23(42), I49-I85. http://dx.doi.org/10.4067/So718-2236201500oroooo8.

Caccia-Bava, A., Feixa, C., \& González, Y. (Orgs.). (2004). Fovens na America Latina. São Paulo: Escrituras.

Cubides, H.J., Laverde, M.C. \& Valderrama, C.E. (eds.) (1998) 'Viviendo a toda'. fóvenes, territorios culturales y nuevas sensibilidades. Bogotá: Fundación Universidad Central.

Duarte, C. (20I5). El adultocentrismo como paradigma y sistema de dominio. Análisis de la reproduccion de imaginarios en la investigación social chilena sobrejuventud (tesis doctoral). Bellaterra: Universitat Autònoma de Barcelona.

Faletto, E. (I986). La juventud como movimiento social. Revista de Estudios de Fuventud, 20: 7I-8I.

Fernández-Planells, A., Feixa, C., \& Figueras, M. (2013). El I5M en España: 
diferencias y similitudes en las prácticas comunicativas con los movimientos previos. Última Década, 2I(39), II5-138. http://dx.doi.org/Io.4067/ So718-22362013000200006.

Feixa, C. (1998). Dejóvenes, bandasy tribus. Barcelona: Ariel. ( $5{ }^{\mathrm{a}}$ Edición Ampliada, 2012).

Feixa, C. (1998b). El reloj de arena. Culturasjuveniles en México. México: Centro de Estudios e Investigaciones sobre la Juventud, Secretaria de Educación Pública.

Feixa, C. (2000). Generación @. La juventud en la era digital. Nómadas, 13, 769 I.

Feixa, C. (2006). Generación XX. Teorías sobre la juventud en la era contemporánea. Revista Latinoamericana de Ciencias Sociales, Niñez y fuventud, 4(2), 2I45 .

Feixa, C. (2009). Generación Replicante. El País, La Cuarta Página, Madrid, Iо/IO/og.

Feixa, C. (2010). El imperio de los jóvenes. Le Monde Diplomatique, I4(180), 24.

Feixa, C. (20II). La generación indignada. El País, La Cuarta Página. Madrid, 29/og/II.

Feixa, C. (Dir.), Porzio, L., \& Recio, C. (Coords.). (2006). Fóvenes latinos en Barcelona. Espacio público y cultura urbana. Barcelona: Anthropos.

Feixa, C., \& Figueras, M. (2018). Emergence of (Hybrid) Youth Cultures. Communication Theory, 28(2), 224-228. https://doi.org/ro.Io93/ct/qtxor3

Feixa, C., \& González, Y. (2005). The Socio-Cultural Construction of Youth in Latin America: Achievements and Failures. In: H. Helve \& G. Holm (Eds.), Contemporary Youth Research: Local Expressions and Global Connections (pp. 24I-256). Burlington and Andershot: Ashgate.

Feixa, C., Costa, C., \& Pallarés, J. (Eds.). (2002). Movimientosjuveniles en la Península Ibérica. Graffitis, grifotas, okupas. Barcelona: Ariel.

Feixa, C., Fernández-Planells, A., \& Figueras, M. (20I6). Generación Hashtag. Los movimientos juveniles en la era de la web social, Revista Latinoamericana de Ciencias Sociales, Niñez y Fuventud, I4(I), I07-I20. http://dx.doi.or$\mathrm{g} /$ Io.II6oo/16927I5X.I4I630III5

Feixa, C., Molina, F., \& Alsinet, C. (Eds.). (2002). Movimientosjuveniles en América Latina. Pachucos, malandros, punketas. Barcelona: Ariel.

Feixa, C., Portillo, M., Urteaga, M., González, Y, \& Aguilera, O. (2012). From Generation X to Generation @. Transitional traces and youth identities in Latin America (pp. 268-292). In: Ch. Henseler (Ed.), Generation X Goes Global. Maping a Youth Culture in Motion. London \& New York: Routledge.

García Canclini, N. (1989). Culturas hibridas. Estrategias para entrary salir de la modernidad. Ciudad de México, México: Grijalbo.

García Canclini, N. (2004). Diferentes, desiguales y desconectados. Mapas de la interculturalidad, Barcelona: Gedisa. 
Hopenhayn, M. (coord) (2007) La juventud en Iberoamérica. Tendencias y urgencias. Buenos Aires: CEPAL-OIJ.

Juventud sin Futuro (20II). Fuventud sinfuturo. Madrid: Popular.

Maffesoli, M. (I99o). El tiempo de las tribus. Barcelona: Icària.

Margulis, M. (Ed.) (1997) Lajuventudes más que una palabra. Buenos Aires: Biblos.

Martín-Barbero, J. (1998). Jóvenes: des-orden cultural y palimpsestos de identidad. En: H. J. Cubides, M. C Laverde, \& C. E. Valderrama (eds.) 'Viviendo a toda', pp. 22-37. Bogotá: Fundación Universidad Central.

Martín-Barbero, J. (Autor), Feixa, C. \& Figueras, M. (2017). Fóvenes, entre el palimpsesto y el hipertexto. Barcelona, España: NED.

Medina, G. (Ed.) (200o). Aproximaciones a la diversidad de lojuvenil. México: El Colegio de México.

Nateras, A. (Ed.) (2002) Fóvenes, culturas eidentidades urbanas. México: UAM.

Leccardi, C., \& Feixa, C. (20II). El concepto de generación en las teorías sobre la juventud. Última Década, I8(34), II-32. http://dx.doi.org/Io.4067/ So718-22362011000100002.

Oliart, P., \& Feixa, C. (2012). Youth studies in Latin America. On social actors, youth policies and new citizenships. Young, 20(4), 329-344.

Pérez Islas, J.A. (2006). Trazos para un mapa de la investigación sobre juventud en América Latina. Papers. Revista de Sociología, (79), I45-I70.

Portillo, M., Urteaga, M., González, Y., Aguilera, O. \& Feixa, C. (2012). De la de Generación X a la Generación @. Trazos transicionales e identidades juveniles en América Latina. Última Década, 20(37), I37-I74. http:// dx.doi.org/10.4067/So718-22362012000200007.

Reguillo, R. (199I) En la calle otra vez. Las bandas: identidad urbana y usos de la comunicación. México: ITESO.

Reguillo, R. (1998). El año dos mil, ética, política y estéticas: imaginarios, adscripciones y prácticas juveniles. En: H. J. Cubides, M. C. Laverde, \& C. E. Valderrama (Eds.). Viviendo a toda. Fóvenes, territorios culturales y nuevas sensibilidades (pp. 57-82). Santa Fe de Bogotá: Siglo del Hombre Editories.

Reguillo, R. (2000). Emergencia de culturasjuveniles. Buenos Aires: Norma.

Reguillo, R. (2005). Los estudios culturales: el mapa incómodo de un relato inconcluso. Redes.com, Revista de estudios para el desarrollo social de la comunicación, 2, I89-199. Recuperado de: https://dialnet.unirioja.es/ servlet/articulo? codigo $=366$ II54.

Reguillo, R. (2012). Culturasjuveniles. Formas políticas del desencanto. Buenos Aires: Siglo Veintiuno Editores.

Reguillo, R. (20I7). Paisajes insurrectos. Fóvenes, redes y revueltas en el otoño civilizatorio. Barcelona, España: NED.

Sarlo, B. (1994). Escenas de la vida postmoderna. Buenos Aires: Ariel. 
Scolari, C. A. (2013). Entrevista a Rossana Reguillo. Digitalismo.com, 3 Julio 2013. Descargado de: http://www.digitalismo.com/2004-20I4/entrevista-a-rossana-reguillo/.

Tapscott, D. (1998). Growing Up Digital: The Rise of the Net Generation. New York: McGraw-Hill.

Unesco. (1983). Lajuventud en la década de los 80. Salamanca: Sígueme.

Urteaga, M. (20II). La construcción juvenil de la sociedad. México: UAM.

Valenzuela, J. M. (1998). Identidades juveniles. In H. J. Cubides, M. C. Laverde, \& C. E. Valderrama (Eds.). Viviendo a toda. Fóvenes, territorios culturales y nuevas sensibilidades (pp. 38-45). Santa Fe de Bogotá: Siglo del Hombre Editories.

Valenzuela, J. M. (Ed.). (20I6). Fuvenicidio. Ayotzinapay las vidas precarias en América Latina. Barcelona: NED. 